OPEN ACCESS

Edited by:

Emmanuel Pinteaux,

The University of Manchester,

United Kingdom

Reviewed by:

Luigi Sironi,

University of Milan, Italy

Laura Castiglioni,

University of Milan, Italy

*Correspondence:

Baohua Cheng

chengbh1979@163.com

$\mathrm{Bo} \mathrm{Bai}$

bbai@mail.jnmc.edu.cn

Specialty section:

This article was submitted to

Stroke,

a section of the journal

Frontiers in Neurology

Received: 21 September 2020 Accepted: 04 December 2020 Published: 23 December 2020

Citation:

Shao Z, Dou S, Zhu J, Wang H, Xu D, Wang C, Cheng B and Bai B (2020) The Role of Mitophagy in Ischemic Stroke. Front. Neurol. 11:608610 doi: 10.3389/fneur.2020.608610

\section{The Role of Mitophagy in Ischemic Stroke}

\author{
Ziqi Shao ${ }^{1}$, Shanshan Dou ${ }^{2}$, Junge Zhu ${ }^{1}$, Huiqing Wang ${ }^{1}$, Dandan $X u^{1}$, Chunmei Wang ${ }^{2}$, \\ Baohua Cheng ${ }^{2 *}$ and $\mathrm{Bo} \mathrm{Bai}^{2 *}$ \\ ${ }^{1}$ Cheeloo College of Medicine, Shandong University, Jinan, China, ${ }^{2}$ Neurobiology Institute, Jining Medical University, Jining, \\ China
}

Mitochondria are important places for eukaryotes to carry out energy metabolism and participate in the processes of cell differentiation, cell information transmission, and cell apoptosis. Autophagy is a programmed intracellular degradation process. Mitophagy, as a selective autophagy, is an evolutionarily conserved cellular process to eliminate dysfunctional or redundant mitochondria, thereby fine-tuning the number of mitochondria and maintaining energy metabolism. Many stimuli could activate mitophagy to regulate related physiological processes, which could ultimately reduce or aggravate the damage caused by stimulation. Stroke is a common disease that seriously affects the health and lives of people around the world, and ischemic stroke, which is caused by cerebral vascular stenosis or obstruction, accounts for the vast majority of stroke. Abnormal mitophagy is closely related to the occurrence, development and pathological mechanism of ischemic stroke. However, the exact mechanism of mitophagy involved in ischemic stroke has not been fully elucidated. In this review, we discuss the process and signal pathways of mitophagy, the potential role of mitophagy in ischemic stroke and the possible signal transduction pathways. It will help deepen the understanding of mitophagy and provide new ideas for the treatment of ischemic stroke.

Keywords: molecular mechanisms, signaling pathway, ischemia-reperfusion injury, ischemic stroke, mitophagy

\section{INTRODUCTION}

Stroke refers to a group of diseases that cause brain tissue damage due to the sudden rupture of blood vessels or the blockage of blood flow into the brain, including hemorrhagic and ischemic stroke. It has the characteristics of high morbidity and high mortality. Ischemic stroke accounts for $\sim 87 \%$ of the total number of stroke patients (1). Ischemic stroke refers to a type of disease in which brain tissue necrosis is caused by a narrowing or occlusion of the blood supply arteries (carotid and vertebral arteries) of the brain and insufficient blood supply to the brain. At present, thrombolysis is considered to be the most important method for the treatment of ischemic stroke (2). However, due to the limitations of current thrombolytic therapy such as an optimal treatment time window of only $4.5 \mathrm{~h}$ (3), enhancing the self-resistance and protection of neurons has become the focus which has attracted more researchers (4).

Mitochondria are where the oxidative metabolism of eukaryotes takes place, and the major producers of intracellular reactive oxygen species (ROS). They can also regulate membrane potential and control programmed cell death (5). Based on the complex structure and important functions of mitochondria, they are closely related to many diseases including ischemic stroke. When ischemic stroke occurs, the dynamic balance maintained by mitochondria is broken, and 
related signaling pathways are activated, which lead to cascade damage to nerve cells (6). In the ischemic period of ischemic stroke, mitochondria cannot synthesize enough ATP or cause energy disorders due to lack of oxygen and energy substances. During the reperfusion period, the increase of ROS and mitochondrial membrane lipid peroxidation lead to oxidative stress damage. Increased ROS also disrupts the calcium pump on the mitochondrial membrane, which induces calcium overload and inflammatory response. In addition to these pathological changes, cell death in ischemic stroke including apoptosis and autophagy are all related to the loss of mitochondrial function (7). Therefore, the research on the correlation between mitochondria and ischemic stroke can not only fully explain the mechanism of the occurrence and development of ischemic stroke, but also provide potential guidance and help for the innovative treatment of ischemic stroke.

Autophagy is a biological process in which organelles and proteins are degraded by lysosomes in eukaryotic cells (8). One of the main functions of autophagy is to keep cells alive when they are threatened by stressful death (9). This is an important evolutionary conservation mechanism for eukaryotic cells to maintain homeostasis and achieve renewal (10). Although autophagy in a broad sense includes macroautophagy, microautophagy and chapeon-mediated autophagy (11), it is commonly referred to as macroautophagy. Mitophagy is a type of macroautophagy by which cells selectively clear impaired or dysfunctional mitochondria through the mechanism of autophagy (12). It plays an important role in mitochondrial quality control and cell survival (13). More and more studies have shown that mitophagy is associated with neurodegenerative diseases such as Parkinson's disease (PD), Alzheimer disease (AD) and Huntington's (HD) and brain injury (14). Although there have been studies showing that mitophagy is closely related to ischemic stroke $(4,15)$, the exact roles of mitophagy still need to be studied further. In this review, we focus on the research progress in the occurrence and regulation of mitophagy in ischemic stroke.

\section{MITOCHONDRIAL DYNAMICS AND MITOPHAGY}

\section{The Molecular Mechanism of Mitochondrial Dynamics}

Mitochondria are highly dynamic organelles that adapt to various stress conditions to meet the energy metabolism and other biological needs through continuous fusion and fission to change their shape (16). This biological process is called mitochondrial dynamics, which is an important basis for maintaining cell homeostasis (17). Mitochondrial fusion is a multi-step process in a certain order: (1) mitochondrial trans-tethering; (2) mitochondrial outer membrane fusion; (3) mitochondrial inner membrane fusion (16). The fusion process of mitochondria is mainly completed by activating three GTPases: mitofusins 1(Mfn1), mitofusins 2 (Mfn2) and optic atrophy 1 (OPA1) (18). Among them, Mfn1 and Mfn2 mainly mediate mitochondrial outer membrane fusion, and
OPA1 mainly participates in the mitochondrial inner membrane fusion process (19). The fission process is mainly mediated by dynamin-related protein 1 (Drp1) and fission protein 1 (Fis1) (18). Under the stimulus, Fis1 mediates the translocation of Drp1 in the cytoplasm to the outer mitochondrial membrane. Drp1 accumulates at the mitochondrial fission site to form a "ring" structure and then combines with Fis1 to form a complex, which is gradually compressed until the mitochondria ruptures. Finally, two independent mitochondria are produced (20) (Figure 1).

Disturbance of mitochondrial dynamics is an important phenomenon in cerebral ischemia/reperfusion injury. Studies have found that there is a loss of OPA1 complex during reperfusion (21). In the rat model of cerebral ischemia/reperfusion injury, the expression of Mfn2 in the cerebral cortex is significantly reduced (22). Research has indicated that activating Mfn1 could reduce cerebral ischemia/reperfusion injury (23). In addition, the fission of mitochondria in the hippocampus is found to be activated, and the mitochondria become more and more fragmented with time (24). Previous studies have shown that inhibiting Drp1dependent mitochondrial fission could protect against cerebral ischemia/reperfusion injury (25). Similarly, the inhibition of Fis1 could also achieve the protective effect (26).

\section{Interplay Between Mitochondrial Dynamics and Mitophagy}

Mitochondrial fusion could repair slightly damaged mitochondria. And mitochondrial fission could not only achieve normal number of proliferation, but also selectively distribute the damaged components of mitochondria to the offspring, which result in healthy mitochondria and severely damaged mitochondria (27). The membrane potential of severely damaged mitochondria cannot be restored. Therefore, the severely damaged mitochondria are unable to participate in fusion, which will be cleared through mitophagy (28). The interaction and mutual regulation between mitochondrial dynamics and mitophagy are important mechanisms for maintaining mitochondrial homeostasis and ensuring mitochondrial quality. In the early stage of ischemia, Drp1dependent mitophagy is found to contribute to the clearance of damaged mitochondria (29). Studies have shown that mitochondrial fragmentation could regulate mitophagy and apoptosis in cerebral ischemia/reperfusion injury (30). It is also found that OPA1 and Mfn2 are reduced in cerebral ischemia, thereby inducing mitophagy (31). These findings indicate that mitochondrial dynamics is closely related to mitophagy in cerebral ischemia/reperfusion injury.

\section{Mechanisms and Regulatory Pathways of Mitophagy} The Process of Mitophagy

The process of mitophagy is similar to ordinary autophagy. First, permeability changes occur after mitochondria are damaged, which leads to mitochondrial depolarization, and induces the activation of mitophagy-related proteins. Then, the isolation membrane wraps the damaged mitochondria and 


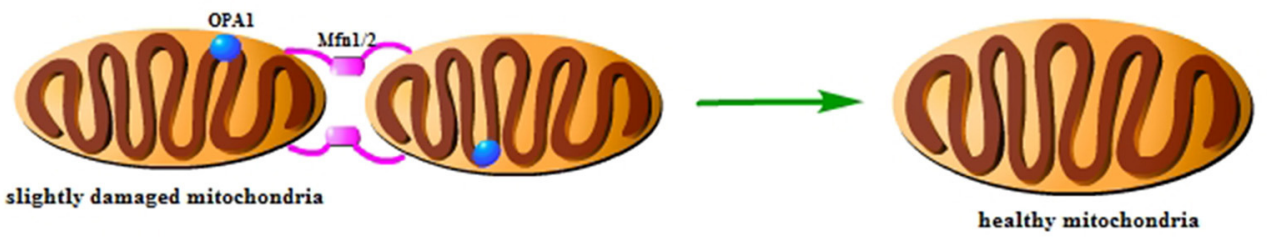

B

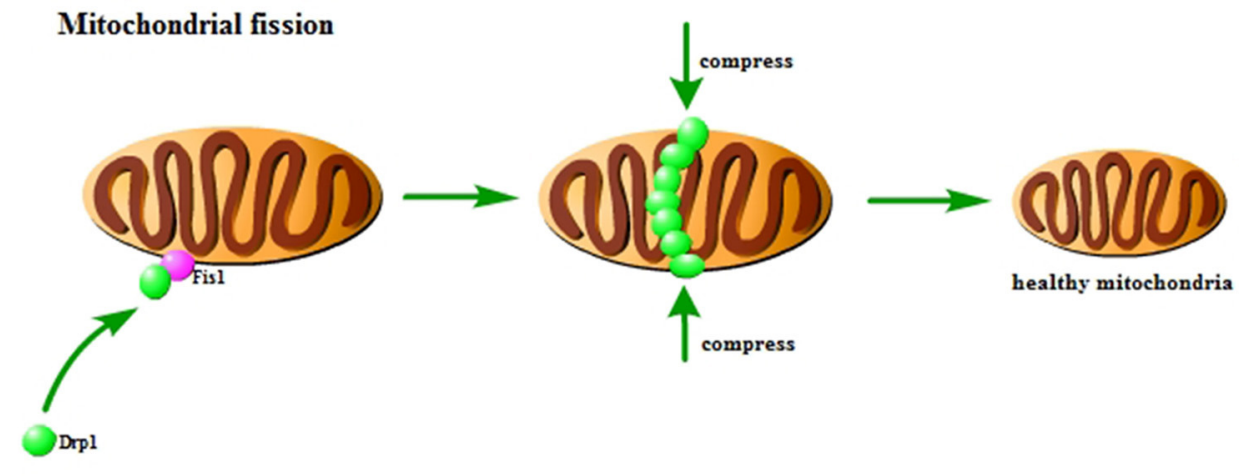

Cytoplasm

FIGURE 1 | Pattern diagram of mitochondrial fusion and fission. (A) Mitochondrial fusion. The Mfn1/2 interaction causes the outer membrane to fuse, and then the Opa1 interaction causes the inner membrane to fuse. It could repair slightly damaged mitochondria. (B) Mitochondrial fission. Fis1 on the mitochondria recruits Drp1 to the mitochondria to form a finger ring structure, which squeezes and ruptures the mitochondria. It could distribute the damaged components of mitochondria to the offspring. The severely damaged mitochondria will be cleared by mitophagy.

forms mitophagosomes. After the formation of mitophagosomes, fusion with lysosomes to form mitolysosomes results in the degradation of damaged mitochondria. This process requires the participation of microtubule-associated protein light chain 3 (LC3) and the linker proteins p62, NBR1 and optineurin connecting mitochondria and LC3. In addition, Nix/BNIP3, FUNDC1 also play an important role in this process (32).

\section{Mitophagy Signaling Pathway}

The mechanisms of mitophagy in cells mainly include Parkindependent pathways and Parkin-independent pathways. There are multiple signals involved in the regulation of mitophagy, as showed in Figure 2. The current review focuses on PINK1/Parkin, BNIP3/NIX, and FUNDC1 pathway.

\section{Parkin-Dependent Mitophagy Pathway}

At the beginning of the 21st century, the laboratory of Richard Youle found that Parkin, an E3 ubiquitin ligase, could mediate mitochondria to be wrapped by autophagosomes, which creates a new breakthrough for the study of mitophagy (33). Then, subsequent research found that PINK1 (phosphatase and tensin homolog (PTEN)-induced putative protein kinase (1), a serine/threonine kinase, is located upstream of Parkin (34-36). PINK1 can phosphorylate Parkin and promote the translocation of Parkin from cytoplasm to mitochondria
(37). PINK1/Parkin is the clearest pathway for the research of mitophagy.

In healthy mitochondria, the PINK1 protein exists on the outer mitochondrial membrane. It can be introduced into the mitochondrial membrane space and degraded by proteases on the inner mitochondrial membrane to maintain the basic level (38). However, when mitochondria are damaged and depolarized, their ability to degrade PINK1 is weakened, and PINK1 can stably exist on the outer mitochondrial membrane (39). Then, it can phosphorylate both ubiquitin and Parkin to recruit Parkin from the cytoplasm to the outer mitochondrial membrane (40). The stability of PINK1 on the outer mitochondrial membrane is necessary for Parkin to be recruited to damaged mitochondria and to stimulate mitophagy. Activated Parkin can ubiquitinate the voltagedependent anion channels 1 (VDAC1) of damaged mitochondria. Then, Parkin is recognized and bound by the signal adaptor protein p62/SQSTM1. P62 could recruit ubiquitinated substances into autophagosomes by binding to LC3, which ultimately leads to mitochondria degraded by lysosome (41-43). In the penumbra of rat cortex, fluorescence results show that PINK1 accumulates on the outer mitochondrial membrane and Parkin mitochondrial translocation occurs following ischemia and reperfusion, and the levels of other related autophagy proteins such as LC3 and Beclin1 are elevated. These results 


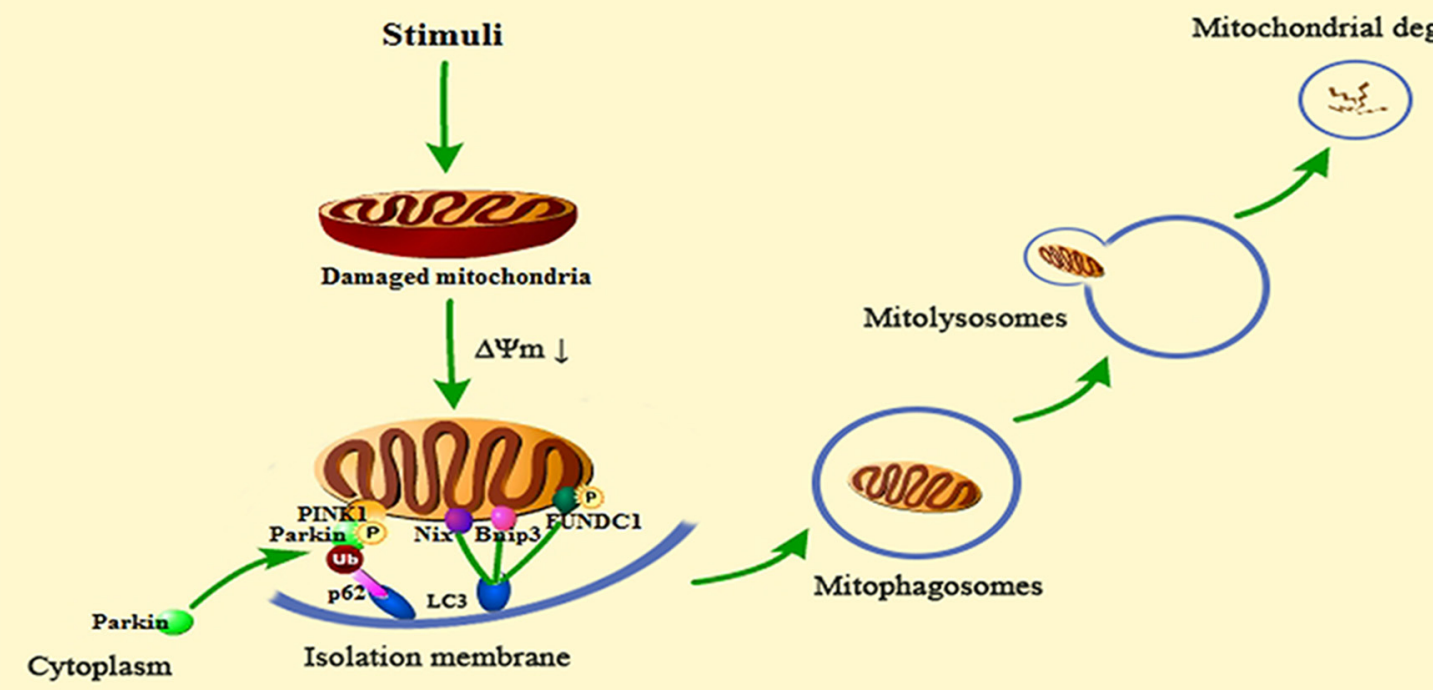

FIGURE 2 | Summary of the mitophagy signaling pathway. When the cell is stimulated, the mitochondria will be damaged. Then the mitochondrial transmembrane potential $(\Delta \Psi \mathrm{m})$ drops, and mitophagy will be activated. PINK1 accumulates on the outer mitochondrial membrane, phosphorylates Parkin and recruits Parkin from the cytoplasm to the mitochondria. Activated Parkin can ubiquitinate the voltage-dependent anion channels 1 (VDAC1) of damaged mitochondria, Then, Parkin is recognized and bound by the signal adaptor protein p62/SQSTM1. P62 could recruit ubiquitinated substances into autophagosomes by binding to microtubule-associated protein light chain 3 (LC3). Bnip3, Nix and phosphorylated FUNDC1 can also connect to LC3 to promote mitophagy. Subsequent mitophagosomes are formed, which bind to lysosomes, and finally lyse damaged mitochondria.

may demonstrate that mitophagy is activated in ischemic stroke (44).

\section{Parkin-Independent Mitophagy Pathway}

Different from PINK1/Parkin-mediated mitophagy, some proteins on the outer mitochondrial membrane can directly recognize and bind LC3. Then, targeted mitochondria are connected with autophagic vesicles, which directly induces mitophagy (45). In this review, we focus on the Nix/Bnip3 and FUNDC1 signaling pathways. They are the most important pathways in Parkin-independent mitophagy.

\section{Nix/Bnip3-Mediated Mitophagy Pathway}

Bnip3 and Nix (BNIP3L) have about 56\% amino acid sequence identity, and both are located in mitochondria (46). Bnip3 is a pro-apoptotic mitochondrial protein. And it is also an important participant in the process of autophagy and even mitophagy (47, 48). Bnip 3 is the target gene of HIF1 $\alpha$ (hypoxia inducible factor $1 \alpha)$. Under hypoxic conditions, Bnip3 could activate autophagy (49). Similarly, in ischemia and reperfusion, Bnip3 could clear damaged mitochondria by activating mitophagy (49, 50). In the process of red blood cell maturation and development, Nix is essential for the removal of mitochondria. Mitochondrial depolarization, increased production of ROS and hypoxia can induce Nix to regulate mitophagy $(51,52)$. Bnip3 and Nix could competitively bind to the anti-apoptotic Bc1-2, dissociate the Bc1-Beclin1 complex and release Beclin1, and then activate autophagy and mitophagy (53). In cerebral ischemia/reperfusion injury, Bnip3 and Nix could participate in the induction of mitophagy (54). However, Studies have found that the upregulation of Nix cannot restore the mitophagy defect caused by Bnip3 deletion in stroke (55). This may indicate that Bnip3 could activate excessive mitophagy leading to cell death, whereas Nix may only regulate basal levels of mitophagy in physiological conditions. Therefore, Bnip3 may be a potential target for the treatment of ischemic stroke in the future.

\section{FUNDC1-Mediated Mitophagy Pathway}

FUNDC1 is a tertiary transmembrane protein on the outer mitochondrial membrane. The FUNDC1 protein contains a Nterminal LC3 interaction region motif, which plays an important role in mitophagy (56). Under normal conditions, FUNDC1 can stably exist on the outer mitochondrial membrane without mediating mitophagy. When mitochondria are damaged or dysfunctional, the affinity of FUNDC1 and LC3 will increase. Then FUNDC1 can be dephosphorylated to activate, which will induce mitophagy (57). In myocardial ischemia/reperfusion, studies have found that hypoxic preconditioning could induce 
FUNDC1-dependent mitophagy to resist ischemia/reperfusion injury (58). However, some studies have shown that inhibition of mitophagy mediated by the mTORC1-ULK1-FUNDC1 pathway can protect myocardium from ischemia/reperfusion injury (59). This indicates that similar mechanisms may exist in cerebral ischemia/reperfusion.

\section{Roles of Mitophagy in Ischemic Stroke}

The brain is the main organ of energy metabolism, and the content of mitochondria in the brain is much higher than other tissues (60). Even if short-term ischemia and hypoxia may cause serious injury to the brain, overproduction of free radicals and calcium overload after reperfusion also cause more extensive damage to cells and tissues of the brain. Current research suggests that cerebral ischemia/reperfusion injury is related to the production of free radicals, excitatory amino acid toxicity, mitochondrial dysfunction, and activation of apoptosis-related genes $(7,61-63)$. Mitochondrial dysfunction is an important part of cerebral ischemia/reperfusion injury, and mitophagy plays a significant role in it. Elimination of abnormal mitochondria through mitophagy is essential for maintaining normal cell function in ischemic stroke.

More than 50 years ago, transmission electron microscopy first discovered the existence of autophagosomes (64-66). As the research on autophagy gets deeper, research methods on autophagy are becoming more and more abundant. Accumulated evidence indicated that autophagy is activated in brain tissue in many nervous system diseases including ischemic stroke (67-70). And mitophagy, as a special type of autophagy, is also found to be activated in ischemic stroke (44). In cerebral ischemia/reperfusion injury, early ischemia and hypoxia damage the structure and function of mitochondria in brain cells. After the oxygen supply and energy supply are restored, the mitochondrial permeability transition pore opens (mPTP) and the mitochondrial membrane potential (MMP) decreases, and then mitochondrial damage is followed by activation of mitochondrial autophagy (71).

Like macroautophagy, we are not sure whether mitophagy is beneficial or harmful in ischemic stroke. The degree of mitochondrial permeability transition (MPT) may play an important role in it (72). Under mild starvation or hypoxia, limited MPT can only damage a small part of mitochondria and then activate mitophagy. At this time, mitophagy not only provides energy by degrading proteins, but also removes damaged mitochondria to protect the cells. In the case of severe starvation or hypoxia, mitophagy is insufficient to clear the damaged mitochondria, and then the autophagy system will be overloaded, which will activate apoptosis-related regulatory proteins and promote the occurrence of apoptosis. When excessive stress causes drastic changes in the MPT of all mitochondria in the cell, cell necrosis will occur.

\section{Neuronal Mitophagy in Ischemic Stroke Enhancing Mitophagy Reduced Cerebral Ischemic-Reperfusion Injury}

Studies have shown that rapamycin could protect against cerebral ischemia/reperfusion injury by activating mitophagy and reducing mitochondrial dysfunction in transient middle cerebral artery occlusion (tMCAO) model. And these protective effects can be reversed by 3-methyladenine, an autophagy inhibitor (73). There are similar findings in the oxygen-glucose deprivation model of hippocampal neurons (74). And activating mitophagy to clear excessively aggregated and damaged mitochondria can reduce neuronal damage caused by cerebral ischemia/reperfusion injury (75-79). Knockout of the mitophagy-related gene Bnip3L could aggravate cerebral ischemia/reperfusion injury, and overexpression of this gene could rescue (54). Studies have also found that activating Parkin-dependent mitophagy could inhibit the activation of NLRP3 inflammasome to reduce cerebral ischemia/reperfusion injury (80). Activation of PARK2mediated mitophagy may be the basis for protecting endoplasmic reticulum stress in cerebral ischemia/reperfusion injury (81) and extending the limited reperfusion window (82).

\section{Inhibiting Mitophagy Reduced Cerebral Ischemic-Reperfusion Injury}

However, there are still some studies showing that inhibiting excessive mitophagy can play a protective role in cerebral ischemia/reperfusion injury. In the middle cerebral artery occlusion model (MCAO) of ischemic stroke, studies have found that inhibiting mitophagy can protect against cerebral ischemia/reperfusion injury (83). In the oxygen glucose deprivation model of SH-SY5Y cells, inhibition of mitochondrial calcium uniporter and the influx of $\mathrm{Ca}^{2+}$ into mitochondria could inhibit excessive mitophagy and reduce neuronal damage (84). And inhibiting Peroxynitrite-mediated mitochondrial activation could reduce neuronal damage in ischemic stroke $(85,86)$. In neuronal death caused by chronic cerebral hypoperfusion, it is also found that inhibiting excessive mitophagy could exert neuroprotective effects (87). Similarly, inhibition of AMPK-mediated mitophagy could reduce the ischemic and hypoxic damage of neurons in ischemic hypoxic encephalopathy (88).

Although the differences in the above results may be caused by different ischemia or reperfusion time, different cell types, or even different experimental environments, it is undeniable that mitophagy plays an important role in the pathological mechanism of cerebral ischemia/reperfusion injury. When it is at the basic level, it may be beneficial to cell homeostasis and neuron survival. But it can be harmful when it reaches excess or deficiency. Therefore, the role of mitophagy in ischemic stroke should be studied more deeply to provide novel ideas and targets for clinical treatment.

\section{Glial Mitophagy in Ischemic Stroke}

Glial cells can not only support, nourish, and protect neurons, but also receive signals from neurons. Through their own function, metabolism and morphological changes, glial cells could affect the function and activity of neurons (89). After cerebral ischemic injury, glial cells are activated. In the early stage of cerebral ischemic injury, the activation of glial cells can play a certain neuroprotective effect, but the excessive activation of glial cells can produce a series of inflammatory factors or mediators to mediate neuronal degeneration (90). Previous study has found 
that hypoxia and reoxygenation of astrocytes caused increased mitochondrial fission and mitophagy (91). In the rat cortex after cerebral ischemia and reperfusion, the activation of mitophagy in astrocytes is also found (83). There are many studies on glial cells autophagy in ischemic stroke while mitophagy-related reports are few. More investigations on astrocytes or microglial mitophagy are needed.

\section{Interplay Between Mitophagy and Other Cellular Processes in Ischemic Stroke}

Mitochondria are the most important organelles involved in energy metabolism in cells. They play a key role in cell signal transduction, free radical generation and apoptosis induction, and determine the survival and death of cells. In the occurrence and development of ischemic stroke, mitophagy is closely related to many biological processes in the cell, such as apoptosis, oxidative stress, and inflammation (Figure 3). These biological processes interact with mitophagy to regulate mitochondrial quality, which could affect the survival and death of nerve cells.

\section{Mitophagy and Apoptosis}

Unlike mitophagy, apoptosis only has a one-way effect on cell fate. It removes aging and severely damaged cells through a programmed death regulation mechanism. Mitophagy and apoptosis have obvious differences in biochemical metabolic pathways and morphology, but they are functionally antagonistic, coordinated, and promote each other, and participate in the regulation of mitochondrial quality (92-95).

Mitophagy and apoptosis are mostly mutually antagonistic to achieve mutual regulation. Under stress conditions such as ischemia and hypoxia, the phosphorylation of the anti-apoptotic protein $\mathrm{Bcl}-2$ could destroy its binding to autophagy-related protein Beclin1 and activate mitophagy. At the same time, Bcl-2 could prevent the release of pro-apoptotic proteins by maintaining the integrity of the mitochondrial membrane, which finally inhibits the occurrence of cell apoptosis (96). When the cell is under continuous and severe stress, apoptosis can inhibit the occurrence of mitophagy by cleaving the key autophagy protein Beclin 1 by activated Caspase and avoid the mitochondrial dysfunction caused by excessive mitophagy $(97,98)$. However, other studies have shown that mitophagy and apoptosis are functionally coordinated and mutually promoted. Excessive induction of mitophagy can cause the leakage of cathepsin and other hydrolytic enzymes in lysosomes or autophagic lysosomes, and promote the occurrence of apoptosis (99).

Studies have reported that activating mitophagy could inhibit cell apoptosis in ischemic stroke. During the reperfusion phase, mitophagy could inhibit neuronal apoptosis by removing damaged mitochondria (100), and remote ischemic post conditioning could promote Parkin/DJ-1-mediated mitophagy to attenuate apoptosis in MCAO rats (101). Similarly, enhancing Parkin /PINK1-mediated mitophagy could inhibit apoptosis caused by cerebral ischemia/reperfusion injury in hippocampal neurons (74). However, there are still some studies indicating that inhibiting excessive mitophagy can reduce apoptosis. Mitophagy-related protein Bnip3 and Nix could induce excessive mitophagy to promote cell apoptosis in ischemic stroke (55). And inhibition of PINK1/Parkin-mediated mitophagy could reduce the number of apoptotic cells in the cortex of the model group in cerebral ischemia/reperfusion injury (86).

\section{Mitophagy and Oxidative Stress}

Oxidative stress is caused by free radicals to produce oxidative damage to deoxyribonucleic acid (DNA), lipids and proteins, which leads to aging and neurodegenerative diseases. The oxidation of proteins, lipids and DNA are all related to ROS (102). ROS can lead to mitochondrial lipid peroxidation, membrane potential collapse and ATP synthesis disorder. Mitochondria are the main source of ROS production (103). Mitophagy is closely related to oxidative stress. And it also plays a dual role in oxidative stress.

Under physiological conditions, low levels of ROS can usually be degraded by some antioxidant enzymes or substances. Maintaining the balance between ROS production and degradation is very important for the normal physiological functions. If the anti-oxidant substances in the cell cannot effectively degrade ROS, it will make ROS accumulate in the cell and then cause oxidative stress (104). Excessive ROS and other substances can preferentially activate mitophagy, allowing it to selectively degrade damaged mitochondria, which could reduce damage to cells (105). There are studies showing that activating mitophagy could reduce oxidative stress damage in ischemia/reperfusion injury. In renal ischemia/reperfusion injury, ischemic preconditioning can protect mitochondrial function by activating mitophagy and inhibit the production of ROS (106), and activation of ROS-dependent autophagy promotes the survival of liver endothelial cells in liver ischemia/reperfusion injury (107). Also, in cerebral ischemia/reperfusion injury, enhancing mitophagy could reduce excessive accumulation of ROS (74). However, mitophagy also can directly induce the death of oxidized cells. In myocardial ischemia/reperfusion injury, inhibiting PINK1/Parkin-mediated mitophagy could reduce ROS production and protect against neurons damage (108). Likely, there may be a similar mechanism in cerebral ischemia/reperfusion injury.

\section{Mitophagy and Inflammation}

Inflammation is an important self-defense mechanism of the body, and mitochondria play a significant role in the occurrence and development of inflammation (109). After being stimulated by factors such as infection, trauma, lipopolysaccharide, high temperature, and hypoxia, the body's innate immune cells activate and trigger an inflammatory response. Under the stimulation of inflammatory factors, ROS and other inflammatory mediators released by neutrophils can induce mitochondrial structural and functional damage (110), including decreased activity of electron transport chain complexes, decreased membrane potential, ATP depletion, and decreased mitochondrial DNA. Mitophagy is of great significance for removing damaged mitochondria and maintaining the function of cellular mitochondrial network. It can remove 


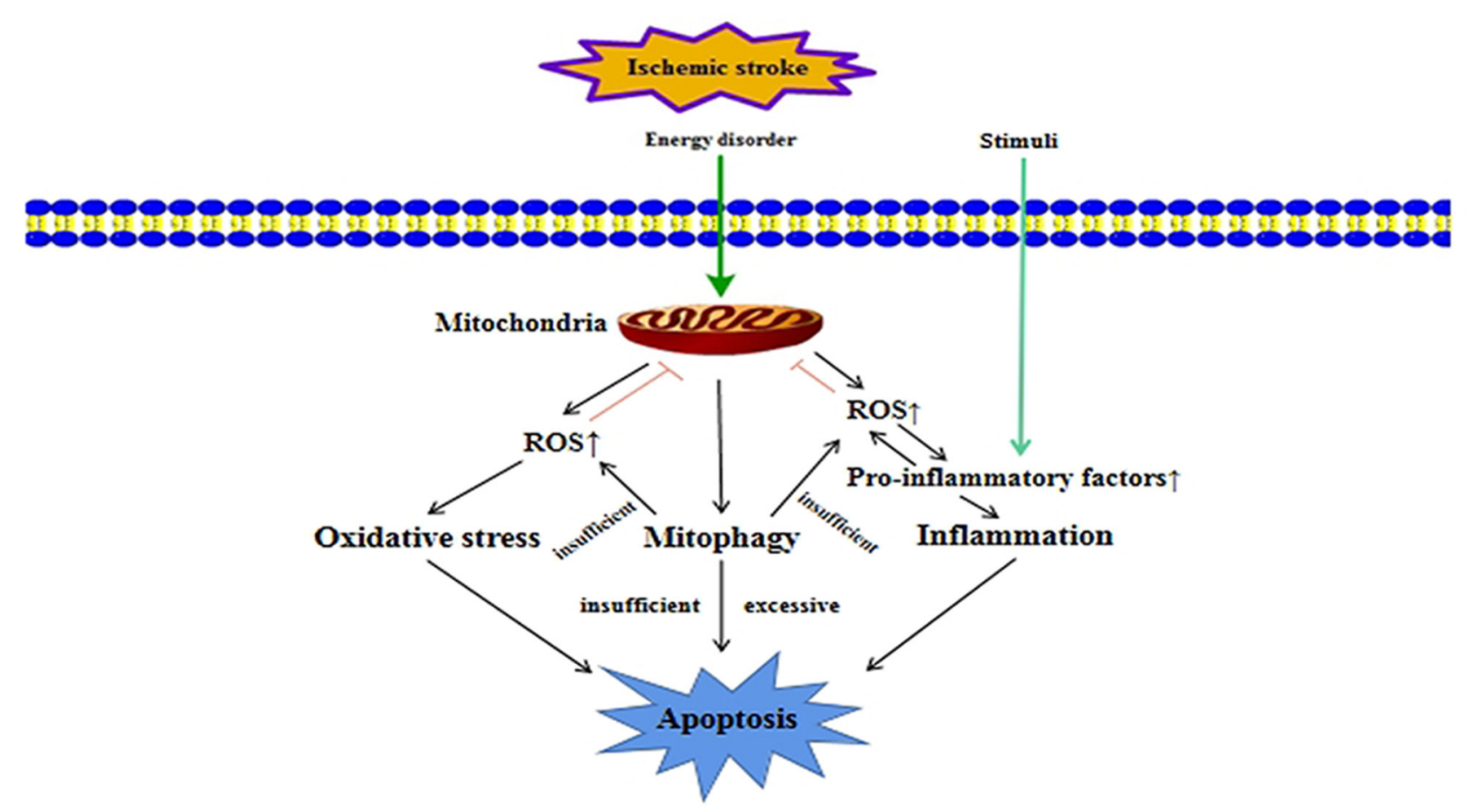

FIGURE 3 | Crosstalk between mitophagy and other cellular processes in ischemic stroke. In ischemic stroke, ischemia and hypoxia can cause energy disorders, which will damage mitochondria. Mitophagy is activated when mitochondria are damaged. When mitophagy is insufficient or excessive, it can lead to apoptosis. Mitochondrial damage will also release a large amount of ROS, leading to oxidative stress damage. At the same time, excessive ROS will promote the release of pro-inflammatory factors, which lead to inflammation. When mitophagy is not enough to clear over-produced ROS, it will aggravate oxidative stress damage and inflammation, which could promote apoptosis.

damaged mitochondria, promote healthy mitochondrial proliferation and other processes, improve mitochondrial homeostasis and function, and exert anti-inflammatory effects (111).

In recent years, a large number of studies have shown that autophagy is inhibited or weakened in inflammatory diseases, and the body is manifested as excessive inflammation or excessive activation of inflammasomes (112-114). And the related mechanisms of mitophagy and inflammation have also been studied in depth $(110,115)$. In cerebral ischemia/reperfusion injury, the relationship between NLRP3 inflammasome activation and mitophagy has been the most studied. In the rat model of ischemic stroke, studies have found that Parkin-dependent mitophagy could effectively inhibit the activation of NLRP3 inflammasome (80). And in myocardial ischemia/reperfusion injury, activation of PINK1/Parkin-mediated mitophagy could reduce cell apoptosis and inflammatory response (116). At present, the interaction and relationship between the specific mechanisms and pathways of mitophagy and inflammation in ischemic stroke are not fully understood. Uncovering the complex regulatory network mechanism between them can provide a theoretical basis for finding new treatment methods of ischemic stroke.

\section{CONCLUSION AND PROSPECTS}

Obviously, mitophagy plays an important role in ischemic stroke through many regulatory factors and other related cellular processes. Although the role of mitophagy has not been unified yet, most studies have proved that in cerebral ischemia/reperfusion injury, mitophagy as an early defense mechanism could clear damaged mitochondria in time, thereby reducing the further damage to normal mitochondria caused by stimulation. However, when the process of mitophagy is blocked or excessive it will aggravate cerebral ischemia/reperfusion injury. Mitophagy may have different effects on neurons with changes in different pathological stages of ischemia and reperfusion, but the reasons for this change have not been clearly studied. These mechanisms need to be further investigated.

This review summarizes the occurrence and development of mitophagy, the related regulatory factors and signal pathways of mitophagy, and the correlation between mitophagy and other cellular processes after cerebral ischemia/reperfusion, which could help to discover new treatment targets and strategies for ischemic stroke. There are still controversies about the mechanism of mitophagy in cerebral ischemia/reperfusion injury. Exploring mitophagy and its regulation mechanism in cerebral ischemia/reperfusion injury will help to 
grasp the relationship between mitophagy and cerebral ischemia/reperfusion injury and various diseases, and provide new ideas for clinical treatment. The role of mitophagy in the different stages and cells of cerebral ischemia/reperfusion and the reasons for this change, from beneficial to harmful, need to be further studied.

\section{AUTHOR CONTRIBUTIONS}

ZS wrote the manuscript and generated the figures. SD helped to design the figures. JZ, HW, and DX proposed suggestions for

\section{REFERENCES}

1. Benjamin EJ, Muntner P, Alonso A, Bittencourt MS, Callaway CW, Carson AP, et al. Heart disease and stroke statistics-2019 update: a report from the American heart association. Circulation. (2019) 139:e56-e528. doi: 10.1161/CIR.0000000000000659

2. Berkowitz AL, Mittal MK, McLane HC, Shen GC, Muralidharan R, Lyons JL, et al. Worldwide reported use of IV tissue plasminogen activator for acute ischemic stroke. Int J Stroke. (2014) 9:349-55. doi: 10.1111/ijs.12205

3. Ferrell AS, Britz GW. Developments on the horizon in the treatment of neurovascular problems. Surg Neurol Int. (2013) 4:S31-7. doi: 10.4103/2152-7806.109194

4. Guan R, Zou W, Dai X, Yu X, Liu H, Chen Q, et al. Mitophagy, a potential therapeutic target for stroke. J Biomed Sci. (2018) 25:87. doi: 10.1186/s12929-018-0487-4

5. Oyewole AO, Birch-Machin MA. Mitochondria-targeted antioxidants. FASEB J. (2015) 29:4766-71. doi: 10.1096/fj.15-275404

6. Ham PB 3rd, Raju R. Mitochondrial function in hypoxic ischemic injury and influence of aging. Prog Neurobiol. (2017) 157:92-116. doi: 10.1016/j.pneurobio.2016.06.006

7. Yang JL, Mukda S, Chen SD. Diverse roles of mitochondria in ischemic stroke. Redox Biol. (2018) 16:263-75. doi: 10.1016/j.redox.2018.03.002

8. Menzies FM, Fleming A, Rubinsztein DC. Compromised autophagy and neurodegenerative diseases. Nat Rev Neurosci. (2015) 16:345-57. doi: 10.1038/nrn3961

9. Ravanan P, Srikumar IF, Talwar P. Autophagy: the spotlight for cellular stress responses. Life Sci. (2017) 188:53-67. doi: 10.1016/j.lfs.2017.08.029

10. Bialik S, Dasari SK, Kimchi A. Autophagy-dependent cell death-where, how and why a cell eats itself to death. J Cell Sci. (2018) 131:jcs.215152. doi: $10.1242 /$ jcs. 215152

11. Galluzzi L, Green DR. Autophagy-independent functions of the autophagy machinery. Cell. (2019) 177:1682-99. doi: 10.1016/j.cell.2019.05.026

12. Tolkovsky AM. Mitophagy. Biochim Biophys Acta. (2009) 1793:1508-15. doi: 10.1016/j.bbamcr.2009.03.002

13. Pickles S, Vigie P, Youle RJ. Mitophagy and quality control mechanisms in mitochondrial maintenance. Curr Biol. (2018) 28:R170-85. doi: 10.1016/j.cub.2018.01.004

14. Fivenson EM, Lautrup S, Sun N, Scheibye-Knudsen M, Stevnsner T, Nilsen $\mathrm{H}$, et al. Mitophagy in neurodegeneration and aging. Neurochem Int. (2017) 109:202-9. doi: 10.1016/j.neuint.2017.02.007

15. Tang YC, Tian HX, Yi T, Chen HB. The critical roles of mitophagy in cerebral ischemia. Protein Cell. (2016) 7:699-713. doi: 10.1007/s13238-016-0307-0

16. Garone C, Minczuk M, Tilokani L, Nagashima S, Paupe V, Prudent J. Mitochondrial dynamics: overview of molecular mechanisms. Essays Biochem. (2018) 62:341-60. doi: 10.1042/ebc20170104

17. Whitley BN, Engelhart EA, Hoppins S. Mitochondrial dynamics and their potential as a therapeutic target. Mitochondrion. (2019) 49:269-83. doi: 10.1016/j.mito.2019.06.002

18. Chan DC. Mitochondria: dynamic organelles in disease, aging, and development. Cell. (2006) 125:1241-52. doi: 10.1016/j.cell.2006.06.010 revisions. $\mathrm{CW}$ revised the manuscript. $\mathrm{BB}$ and $\mathrm{BC}$ designed the content. All authors read and approved the final manuscript.

\section{FUNDING}

The study was supported by the National Natural Science Foundation of China, Nos. 81870948 (to BB), 81671276 (to BC), 81501018 (to CW) the Natural Science Foundation of Shandong Province of China, No. ZR2014HL040 (to BC); Program Supporting Foundation for Teachers' Research of Jining Medical University of China, No. JYFC2018KJ003 (to SD).

19. Otera H, Ishihara N, Mihara $\mathrm{K}$. New insights into the function and regulation of mitochondrial fission. Biochim Biophys Acta. (2013) 1833:125668. doi: 10.1016/j.bbamcr.2013.02.002

20. Yoon Y, Krueger EW, Oswald BJ, McNiven MA. The mitochondrial protein hFis1 regulates mitochondrial fission in mammalian cells through an interaction with the dynamin-like protein DLP1. Mol Cell Biol. (2003) 23:5409-20. doi: 10.1128/mcb.23.15.5409-5420.2003

21. Kumar R, Bukowski MJ, Wider JM, Reynolds CA, Calo L, Lepore B, et al. Mitochondrial dynamics following global cerebral ischemia. Mol Cell Neurosci. (2016) 76:68-75. doi: 10.1016/j.mcn.2016.08.010

22. Klacanova K, Kovalska M, Chomova M, Pilchova I, Tatarkova Z, Kaplan P, et al. Global brain ischemia in rats is associated with mitochondrial release and downregulation of $\mathrm{Mfn} 2$ in the cerebral cortex, but not the hippocampus. Int J Mol Med. (2019) 43:2420-8. doi: 10.3892/ijmm.2019.4168

23. Gao J, Wang H, Li Y, Li W. Resveratrol attenuates cerebral ischaemia reperfusion injury via modulating mitochondrial dynamics homeostasis and activating AMPK-Mfnl pathway. Int J Exp Pathol. (2019) 100:337-49. doi: 10.1111/iep.12336

24. Owens K, Park JH, Gourley S, Jones H, Kristian T. Mitochondrial dynamics: cell-type and hippocampal region specific changes following global cerebral ischemia. J Bioenerg Biomembr. (2014) 47:13-31. doi: 10.1007/s10863-014-9575-7

25. Flippo KH, Gnanasekaran A, Perkins GA, Ajmal A, Merrill RA, Dickey AS, et al. AKAP1 protects from cerebral ischemic stroke by inhibiting Drp1-dependent mitochondrial fission. J Neurosci. (2018) 38:8233-42. doi: 10.1523/jneurosci.0649-18.2018

26. Wang M-S, Tang Y-N, Zhang G-F, Chen H-L, Sun X-P, Qin W-W, et al. Selective brain hypothermia-induced neuroprotection against focal cerebral ischemia/reperfusion injury is associated with Fis1 inhibition. Neural Regen Res. (2020) 15:903-11. doi: 10.4103/1673-5374.268973

27. Vásquez-Trincado C, García-Carvajal I, Pennanen C, Parra V, Hill JA, Rothermel BA, et al. Mitochondrial dynamics, mitophagy and cardiovascular disease. J Physiol. (2016) 594:509-25. doi: 10.1113/jp271301

28. Dorn GW, Kitsis RN. The mitochondrial dynamismmitophagy-cell death interactome. Circ Res. (2015) 116:167-82. doi: 10.1161/circresaha.116.303554

29. Zuo W, Zhang S, Xia C-Y, Guo X-F, He W-B, Chen N-H. Mitochondria autophagy is induced after hypoxic/ischemic stress in a Drp1 dependent manner: the role of inhibition of Drp1 in ischemic brain damage. Neuropharmacology. (2014) 86:103-15. doi: 10.1016/j.neuropharm.2014.07.002

30. Xu B, Zhu L, Chu J, Ma Z, Fu Q, Wei W, et al. Esculetin improves cognitive impairments induced by transient cerebral ischaemia and reperfusion in mice via regulation of mitochondrial fragmentation and mitophagy. Behav Brain Res. (2019) 372. doi: 10.1016/j.bbr.2019.112007

31. Kumari S, Anderson L, Farmer S, Mehta SL, Li PA. Hyperglycemia alters mitochondrial fission and fusion proteins in mice subjected to cerebral ischemia and reperfusion. Transl Stroke Res. (2012) 3:296-304. doi: 10.1007/s12975-012-0158-9 
32. Yoshii SR, Mizushima N. Autophagy machinery in the context of mammalian mitophagy. Biochim Biophys Acta. (2015) 1853:2797-801. doi: 10.1016/j.bbamcr.2015.01.013

33. Narendra D, Tanaka A, Suen DF, Youle RJ. Parkin is recruited selectively to impaired mitochondria and promotes their autophagy. J Cell Biol. (2008) 183:795-803. doi: $10.1083 /$ jcb.200809125

34. Narendra DP, Jin SM, Tanaka A, Suen DF, Gautier CA, Shen J, et al. PINK1 is selectively stabilized on impaired mitochondria to activate Parkin. PLoS Biol. (2010) 8:e1000298. doi: 10.1371/journal.pbio.1000298

35. Geisler S, Holmstrom KM, Skujat D, Fiesel FC, Rothfuss OC, Kahle PJ, et al. PINK1/Parkin-mediated mitophagy is dependent on VDAC1 and p62/SQSTM1. Nat Cell Biol. (2010) 12:119-31. doi: 10.1038/ncb2012

36. Matsuda N, Sato S, Shiba K, Okatsu K, Saisho K, Gautier CA, et al. PINK1 stabilized by mitochondrial depolarization recruits Parkin to damaged mitochondria and activates latent Parkin for mitophagy. J Cell Biol. (2010) 189:211-21. doi: 10.1083/jcb.200910140

37. Barodia SK, Creed RB, Goldberg MS. Parkin and PINK1 functions in oxidative stress and neurodegeneration. Brain Res Bull. (2017) 133:51-9. doi: 10.1016/j.brainresbull.2016.12.004

38. Rasool S, Soya N, Truong L, Croteau N, Lukacs GL, Trempe JF. PINK1 autophosphorylation is required for ubiquitin recognition. EMBO Rep. (2018) 19:e44981. doi: 10.15252/embr.201744981

39. Guardia-Laguarta C, Liu Y, Lauritzen KH, Erdjument-Bromage H, Martin B, Swayne TC, et al. PINK1 content in mitochondria is regulated by ER-associated degradation. J Neurosci. (2019) 39:7074-85. doi: 10.1523/JNEUROSCI.1691-18.2019

40. Koyano F, Okatsu K, Kosako H, Tamura Y, Go E, Kimura M, et al. Ubiquitin is phosphorylated by PINK1 to activate parkin. Nature. (2014) 510:162-6. doi: $10.1038 /$ nature 13392

41. Georgakopoulos ND, Wells G, Campanella M. The pharmacological regulation of cellular mitophagy. Nat Chem Biol. (2017) 13:136-46. doi: $10.1038 /$ nchembio. 2287

42. Eiyama A, Okamoto K. PINK1/Parkin-mediated mitophagy in mammalian cells. Curr Opin Cell Biol. (2015) 33:95-101. doi: 10.1016/j.ceb.2015.01.002

43. Ashrafi G, Schwarz TL. The pathways of mitophagy for quality control and clearance of mitochondria. Cell Death Differ. (2013) 20:31-42. doi: $10.1038 / \mathrm{cdd} .2012 .81$

44. Lan R, Wu J-T, Wu T, Ma Y-Z, Wang B-Q, Zheng H-Z, et al. Mitophagy is activated in brain damage induced by cerebral ischemia and reperfusion via the PINK1/Parkin/p62 signalling pathway. Brain Res Bull. (2018) 142:63-77. doi: 10.1016/j.brainresbull.2018.06.018

45. Villa E, Marchetti S, Ricci J-E. No parkin zone: mitophagy without parkin. Trends Cell Biol. (2018) 28:882-95. doi: 10.1016/j.tcb.2018.07.004

46. Zhang J, Ney PA. Role of BNIP3 and NIX in cell death, autophagy, and mitophagy. Cell Death Differ. (2009) 16:939-46. doi: 10.1038/cdd.2009.16

47. Chourasia AH, Macleod KF. Tumor suppressor functions of BNIP3 and mitophagy. Autophagy. (2015) 11:1937-8. doi: 10.1080/15548627.2015.1085136

48. Ney PA. Mitochondrial autophagy: origins, significance, and role of BNIP3 and NIX. Biochim Biophys Acta. (2015) 1853:2775-83. doi: 10.1016/j.bbamcr.2015.02.022

49. Liu XW, Lu MK, Zhong HT, Wang LH, Fu YP. Panax notoginseng saponins attenuate myocardial ischemia-reperfusion injury through the HIF1alpha/BNIP3 pathway of autophagy. J Cardiovasc Pharmacol. (2019) 73:929. doi: 10.1097/FJC.0000000000000640

50. Tang C, Han H, Liu Z, Liu Y, Yin L, Cai J, et al. Activation of BNIP3-mediated mitophagy protects against renal ischemia-reperfusion injury. Cell Death Dis. (2019) 10:677. doi: 10.1038/s41419-019-1899-0

51. Ding WX, Ni HM, Li M, Liao Y, Chen X, Stolz DB, et al. Nix is critical to two distinct phases of mitophagy, reactive oxygen species-mediated autophagy induction and Parkin-ubiquitin-p62-mediated mitochondrial priming. J Biol Chem. (2010) 285:27879-90. doi: 10.1074/jbc.M110.119537

52. Jung J, Zhang Y, Celiku O, Zhang W, Song H, Williams BJ, et al. Mitochondrial NIX promotes tumor survival in the hypoxic niche of glioblastoma. Cancer Res. (2019) 79:5218-32. doi: 10.1158/0008-5472.CAN-19-0198

53. Bellot G, Garcia-Medina R, Gounon P, Chiche J, Roux D, Pouyssegur J, et al. Hypoxia-induced autophagy is mediated through hypoxia-inducible factor induction of BNIP3 and BNIP3L via their BH3 domains. Mol Cell Biol. (2009) 29:2570-81. doi: 10.1128/MCB.00166-09

54. Yuan Y, Zheng Y, Zhang X, Chen Y, Wu X, Wu J, et al. BNIP3L/NIX-mediated mitophagy protects against ischemic brain injury independent of PARK2. Autophagy. (2017) 13:1754-66. doi: 10.1080/15548627.2017.1357792

55. Shi RY, Zhu SH, Li V, Gibson SB, Xu XS, Kong JM. BNIP3 interacting with LC3 triggers excessive mitophagy in delayed neuronal death in stroke. CNS Neurosci Ther. (2014) 20:1045-55. doi: 10.1111/cns.12325

56. Liu L, Feng D, Chen G, Chen M, Zheng Q, Song P, et al. Mitochondrial outer-membrane protein FUNDC1 mediates hypoxia-induced mitophagy in mammalian cells. Nat Cell Biol. (2012) 14:177-85. doi: 10.1038/ncb2422

57. Chen G, Han Z, Feng D, Chen Y, Chen L, Wu H, et al. A regulatory signaling loop comprising the PGAM5 phosphatase and CK2 controls receptor-mediated mitophagy. Mol Cell. (2014) 54:362-77. doi: 10.1016/j.molcel.2014.02.034

58. Zhang W, Siraj S, Zhang R, Chen Q. Mitophagy receptor FUNDC1 regulates mitochondrial homeostasis and protects the heart from I/R injury. Autophagy. (2017) 13:1080-1. doi: 10.1080/15548627.2017.1300224

59. Zhou H, Wang J, Zhu P, Zhu H, Toan S, Hu S, et al. NR4A1 aggravates the cardiac microvascular ischemia reperfusion injury through suppressing FUNDC1-mediated mitophagy and promoting Mff-required mitochondrial fission by CK2alpha. Basic Res Cardiol. (2018) 113:23. doi: 10.1007/s00395-018-0682-1

60. Belanger M, Allaman I, Magistretti PJ. Brain energy metabolism: focus on astrocyte-neuron metabolic cooperation. Cell Metab. (2011) 14:724-38. doi: 10.1016/j.cmet.2011.08.016

61. Sun MS, Jin H, Sun X, Huang S, Zhang FL, Guo ZN, et al. Free radical damage in ischemia-reperfusion injury: an obstacle in acute ischemic stroke after revascularization therapy. Oxid Med Cell Longev. (2018) 2018:3804979. doi: $10.1155 / 2018 / 3804979$

62. Malik AR, Willnow TE. Excitatory amino acid transporters in physiology and disorders of the central nervous system. Int J Mol Sci. (2019) 20:5671. doi: 10.3390/ijms20225671

63. Uzdensky AB. Apoptosis regulation in the penumbra after ischemic stroke: expression of pro- and antiapoptotic proteins. Apoptosis. (2019) 24:687-702. doi: 10.1007/s10495-019-01556-6

64. Ashford TP, Porter KR. Cytoplasmic components in hepatic cell lysosomes. J Cell Biol. (1962) 12:198-202. doi: 10.1083/jcb.12.1.198

65. Deter RL, Baudhuin P, De Duve C. Participation of lysosomes in cellular autophagy induced in rat liver by glucagon. J Cell Biol. (1967) 35:C11-6. doi: $10.1083 /$ jcb.35.2.c11

66. Fedorko M. Effect of chloroquine on morphology of cytoplasmic granules in maturing human leukocytes-an ultrastructural study. J Clin Invest. (1967) 46:1932-42. doi: 10.1172/JCI105683

67. Yamamoto A, Yue Z. Autophagy and its normal and pathogenic states in the brain. Annu Rev Neurosci. (2014) 37:55-78. doi: 10.1146/annurev-neuro-071013-014149

68. Bingol B. Autophagy and lysosomal pathways in nervous system disorders. Mol Cell Neurosci. (2018) 91:167-208. doi: 10.1016/j.mcn.2018.04.009

69. Geronimo-Olvera C, Massieu L. Autophagy as a homeostatic mechanism in response to stress conditions in the central nervous system. Mol Neurobiol. (2019) 56:6594-608. doi: 10.1007/s12035-019-1546-X

70. Wang P, Shao BZ, Deng Z, Chen S, Yue Z, Miao CY. Autophagy in ischemic stroke. Prog Neurobiol. (2018) 163-4:98-117. doi: 10.1016/j.pneurobio.2018.01.001

71. Wang Y, Nartiss Y, Steipe B, McQuibban GA, Kim PK. ROS-induced mitochondrial depolarization initiates PARK2/PARKIN-dependent mitochondrial degradation by autophagy. Autophagy. (2012) 8:1462-76. doi: 10.4161 /auto. 21211

72. Mijaljica D, Prescott M, Devenish RJ. Different fates of mitochondria: alternative ways for degradation? Autophagy. (2007) 3:4-9. doi: 10.4161/auto.3011

73. Li Q, Zhang T, Wang J, Zhang Z, Zhai Y, Yang GY, et al. Rapamycin attenuates mitochondrial dysfunction via activation of mitophagy in experimental ischemic stroke. Biochem Biophys Res Commun. (2014) 444:182-8. doi: 10.1016/j.bbrc.2014.01.032

74. Wu X, Li X, Liu Y, Yuan N, Li C, Kang Z, et al. Hydrogen exerts neuroprotective effects on $\mathrm{OGD} / \mathrm{R}$ damaged neurons in rat hippocampal 
by protecting mitochondrial function via regulating mitophagy mediated by PINK1/Parkin signaling pathway. Brain Res. (2018) 1698:89-98. doi: 10.1016/j.brainres.2018.06.028

75. Di Y, He YL, Zhao T, Huang X, Wu KW, Liu SH, et al. Methylene blue reduces acute cerebral ischemic injury via the induction of mitophagy. Mol Med. (2015) 21:420-9. doi: 10.2119/molmed.2015.00038

76. Li F, Tan J, Zhou F, Hu Z, Yang B. Heat shock protein B8 (HSPB8) reduces oxygen-glucose deprivation/reperfusion injury via the induction of mitophagy. Cell Physiol Biochem. (2018) 48:1492-504. doi: $10.1159 / 000492259$

77. Wang $\mathrm{H}$, Chen S, Zhang $\mathrm{Y}, \mathrm{Xu} \mathrm{H}$, Sun H. Electroacupuncture ameliorates neuronal injury by Pink1/Parkin-mediated mitophagy clearance in cerebral ischemia-reperfusion. Nitric Oxide. (2019) 91:23-34. doi: 10.1016/j.niox.2019.07.004

78. Wang W, Xu J. Curcumin attenuates cerebral ischemia-reperfusion injury through regulating mitophagy and preserving mitochondrial function. Curr Neurovasc Res. (2020) 17:113-22. doi: 10.2174/1567202617666200225122620

79. Wu M, Lu G, Lao YZ, Zhang $\mathrm{H}$, Zheng $\mathrm{D}$, Zheng ZQ, et al. Garciesculenxanthone B induces PINK1-parkin-mediated mitophagy and prevents ischemia-reperfusion brain injury in mice. Acta Pharmacol Sin. (2020). doi: 10.1038/s41401-020-0480-9. [Epub ahead of print].

80. He Q, Li Z, Meng C, Wu J, Zhao Y, Zhao J. Parkin-dependent mitophagy is required for the inhibition of ATF4 on NLRP3 inflammasome activation in cerebral ischemia-reperfusion injury in rats. Cells. (2019) 8:897. doi: $10.3390 /$ cells 8080897

81. Zhang X, Yuan Y, Jiang L, Zhang J, Gao J, Shen Z, et al. Endoplasmic reticulum stress induced by tunicamycin and thapsigargin protects against transient ischemic brain injury: involvement of PARK2-dependent mitophagy. Autophagy. (2014) 10:1801-13. doi: 10.4161/auto.32136

82. Shen Z, Zheng Y, Wu J, Chen Y, Wu X, Zhou Y, et al. PARK2-dependent mitophagy induced by acidic postconditioning protects against focal cerebral ischemia and extends the reperfusion window. Autophagy. (2017) 13:473-85. doi: 10.1080/15548627.2016.1274596

83. Lan R, Zhang Y, Wu T, Ma YZ, Wang BQ, Zheng HZ, et al. Xiao-XuMing decoction reduced mitophagy activation and improved mitochondrial function in cerebral ischemia and reperfusion injury. Behav Neurol. (2018) 2018:4147502. doi: 10.1155/2018/4147502

84. Yu S, Zheng S, Leng J, Wang S, Zhao T, Liu J. Inhibition of mitochondrial calcium uniporter protects neurocytes from ischemia/reperfusion injury via the inhibition of excessive mitophagy. Neurosci Lett. (2016) 628:24-9. doi: 10.1016/j.neulet.2016.06.012

85. Feng J, Chen X, Lu S, Li W, Yang D, Su W, et al. Naringin attenuates cerebral ischemia-reperfusion injury through inhibiting peroxynitritemediated mitophagy activation. Mol Neurobiol. (2018) 55:9029-42. doi: $10.1007 / \mathrm{s} 12035-018-1027-7$

86. Feng J, Chen X, Guan B, Li C, Qiu J, Shen J. Inhibition of peroxynitriteinduced mitophagy activation attenuates cerebral ischemia-reperfusion injury. Mol Neurobiol. (2018) 55:6369-86. doi: 10.1007/s12035-017-0859-x

87. Su SH, Wu YF, Wang DP, Hai J. Inhibition of excessive autophagy and mitophagy mediates neuroprotective effects of URB597 against chronic cerebral hypoperfusion. Cell Death Dis. (2018) 9:733. doi: 10.1038/s41419-018-0755-y

88. Cai CC, Zhu JH, Ye LX, Dai YY, Fang MC, Hu YY, et al. Glycine protects against hypoxic-ischemic brain injury by regulating mitochondriamediated autophagy via the AMPK pathway. Oxid Med Cell Longev. (2019) 2019:4248529. doi: 10.1155/2019/4248529

89. Durkee CA, Araque A. Diversity and specificity of astrocyteneuron communication. Neuroscience. (2019) 396:73-8. doi: 10.1016/j.neuroscience.2018.11.010

90. Li L, Stary CM. Targeting glial mitochondrial function for protection from cerebral ischemia: relevance, mechanisms, and the role of microRNAs. Oxid Med Cellu Longev. (2016) 2016:1-11. doi: 10.1155/2016/6032306

91. Quintana DD, Garcia JA, Sarkar SN, Jun S, Engler-Chiurazzi EB, Russell AE, et al. Hypoxia-reoxygenation of primary astrocytes results in a redistribution of mitochondrial size and mitophagy. Mitochondrion. (2019) 47:244-55. doi: 10.1016/j.mito.2018.12.004

92. Cheng Y, Yang JM. Autophagy and apoptosis: rivals or mates? Chin J Cancer. (2013) 32:103-5. doi: 10.5732/cjc.013.10022
93. Kaminskyy VO, Zhivotovsky B. Free radicals in cross talk between autophagy and apoptosis. Antioxid Redox Signal. (2014) 21:86-102. doi: $10.1089 /$ ars.2013.5746

94. Wu HJ, Pu JL, Krafft PR, Zhang JM, Chen S. The molecular mechanisms between autophagy and apoptosis: potential role in central nervous system disorders. Cell Mol Neurobiol. (2015) 35:85-99. doi: 10.1007/s10571-014-0116-z

95. Praharaj PP, Naik PP, Panigrahi DP, Bhol CS, Mahapatra KK, Patra S, et al. Intricate role of mitochondrial lipid in mitophagy and mitochondrial apoptosis: its implication in cancer therapeutics. Cell Mol Life Sci. (2019) 76:1641-52. doi: 10.1007/s00018-018-2990-x

96. Mukhopadhyay S, Panda PK, Sinha N, Das DN, Bhutia SK. Autophagy and apoptosis: where do they meet? Apoptosis. (2014) 19:555-66. doi: 10.1007/s10495-014-0967-2

97. Wirawan E, Vande Walle L, Kersse K, Cornelis S, Claerhout S, Vanoverberghe I, et al. Caspase-mediated cleavage of Beclin-1 inactivates Beclin-1induced autophagy and enhances apoptosis by promoting the release of proapoptotic factors from mitochondria. Cell Death Dis. (2010) 1:e18. doi: 10.1038/cddis.2009.16

98. Choubey V, Cagalinec M, Liiv J, Safiulina D, Hickey MA, Kuum M, et al. BECN1 is involved in the initiation of mitophagy: it facilitates PARK2 translocation to mitochondria. Autophagy. (2014) 10:1105-19. doi: 10.4161/auto.28615

99. Chen Y, Chen HN, Wang K, Zhang L, Huang Z, Liu J, et al. Ketoconazole exacerbates mitophagy to induce apoptosis by downregulating cyclooxygenase-2 in hepatocellular carcinoma. J Hepatol. (2019) 70:66-77. doi: 10.1016/j.jhep.2018.09.022

100. Zhang X, Yan H, Yuan Y, Gao J, Shen Z, Cheng Y, et al. Cerebral ischemia-reperfusion-induced autophagy protects against neuronal injury by mitochondrial clearance. Autophagy. (2013) 9:1321-33. doi: 10.4161/auto. 25132

101. Zhou M, Xia ZY, Lei SQ, Leng Y, Xue R. Role of mitophagy regulated by Parkin/DJ-1 in remote ischemic postconditioning-induced mitigation of focal cerebral ischemia-reperfusion. Eur Rev Med Pharmacol Sci. (2015) 19:4866-71.

102. Yaribeygi H, Panahi Y, Javadi B, Sahebkar A. The underlying role of oxidative stress in neurodegeneration: a mechanistic review. CNS Neurol Disord Drug Targets. (2018) 17:207-15. doi: 10.2174/1871527317666180425122557

103. Diebold L, Chandel NS. Mitochondrial ROS regulation of proliferating cells. Free Radic Biol Med. (2016) 100:86-93. doi: 10.1016/j.freeradbiomed.2016.04.198

104. Angelova PR, Abramov AY. Role of mitochondrial ROS in the brain: from physiology to neurodegeneration. FEBS Lett. (2018) 592:692-702. doi: 10.1002/1873-3468.12964

105. Fan P, Xie XH, Chen CH, Peng X, Zhang P, Yang C, et al. Molecular regulation mechanisms and interactions between reactive oxygen species and mitophagy. DNA Cell Biol. (2019) 38:10-22. doi: 10.1089/dna.2018.4348

106. Livingston MJ, Wang J, Zhou J, Wu G, Ganley IG, Hill JA, et al. Clearance of damaged mitochondria via mitophagy is important to the protective effect of ischemic preconditioning in kidneys. Autophagy. (2019) 15:2142-62. doi: 10.1080/15548627.2019.1615822

107. Bhogal RH, Weston CJ, Velduis S, Leuvenink HGD, Reynolds GM, Davies $\mathrm{S}$, et al. The reactive oxygen species-mitophagy signaling pathway regulates liver endothelial cell survival during ischemia/reperfusion injury. Liver Transpl. (2018) 24:1437-52. doi: 10.1002/lt.25313

108. Feng Y, Madungwe NB, da Cruz Junho CV, Bopassa JC. Activation of $\mathrm{G}$ protein-coupled oestrogen receptor 1 at the onset of reperfusion protects the myocardium against ischemia/reperfusion injury by reducing mitochondrial dysfunction and mitophagy. Br J Pharmacol. (2017) 174:4329-44. doi: 10.1111/bph.14033

109. Green DR, Galluzzi L, Kroemer G. Mitochondria and the autophagyinflammation-cell death axis in organismal aging. Science. (2011) 333:110912. doi: $10.1126 /$ science. 1201940

110. Zhao Y, Huang S, Liu J, Wu X, Zhou S, Dai K, et al. Mitophagy contributes to the pathogenesis of inflammatory diseases. Inflammation. (2018) 41:1590600. doi: 10.1007/s10753-018-0835-2

111. Tschopp J. Mitochondria: sovereign of inflammation? Eur J Immunol. (2011) 41:1196-202. doi: 10.1002/eji.201141436 
112. Matsuzawa-Ishimoto Y, Hwang S, Cadwell K. Autophagy and inflammation. Annu Rev Immunol. (2018) 36:73-101. doi: 10.1146/annurev-immunol-042617-053253

113. Deretic V, Levine B. Autophagy balances inflammation in innate immunity. Autophagy. (2018) 14:243-51. doi: 10.1080/15548627.2017.1402992

114. Joven J, Guirro M, Marine-Casado R, Rodriguez-Gallego E, Menendez JA. Autophagy is an inflammation-related defensive mechanism against disease. Adv Exp Med Biol. (2014) 824:43-59. doi: 10.1007/978-3-319-07320-0_6

115. Gkikas I, Palikaras K, Tavernarakis N. The role of mitophagy in innate immunity. Front Immunol. (2018) 9:1283. doi: 10.3389/fimmu.2018.01283

116. Yao L, Chen H, Wu Q, Xie K. Hydrogen-rich saline alleviates inflammation and apoptosis in myocardial I/Rinjury via PINK-mediated autophagy. Int $J$ Mol Med. (2019) 44:1048-62. doi: 10.3892/ijmm.2019.4264
Conflict of Interest: The authors declare that the research was conducted in the absence of any commercial or financial relationships that could be construed as a potential conflict of interest.

Copyright (c) 2020 Shao, Dou, Zhu, Wang, Xu, Wang, Cheng and Bai. This is an open-access article distributed under the terms of the Creative Commons Attribution License (CC BY). The use, distribution or reproduction in other forums is permitted, provided the original author(s) and the copyright owner(s) are credited and that the original publication in this journal is cited, in accordance with accepted academic practice. No use, distribution or reproduction is permitted which does not comply with these terms. 